\title{
Coverage Probability of Cell-Free Massive MIMO Systems
}

\author{
A. K. Papazafeiropoulos ${ }^{*, \downarrow}$, P. Kourtessis*, M. Di Renzo ${ }^{\dagger}$, S. Chatzinotas ${ }^{\natural}$, J. M. Senior* \\ ${ }^{*}$ Communications and Intelligent Systems Research Group, University of Hertfordshire, Hatfield, U. K. \\ ${ }^{\dagger}$ Laboratoire des Signaux et Systèmes, CNRS, CentraleSupélec, Université Paris Sud, France \\ ${ }^{\natural}$ SnT - securityandtrust.lu, University of Luxembourg, Luxembourg \\ Email: tapapazaf@gmail.com, marco.direnzo@12s.centralesupelec.fr, symeon.chatzinotas@uni.lu.
}

\begin{abstract}
Despite that ongoing and future networks become denser and increasingly irregular, prior works in the area of cell-free (CF) massive multiple-input-multiple-output (mMIMO) systems relied on the strong assumption of uniformly distributed access points (APs). Actually, this randomness was accounted for only during the simulation and not in the analysis. Consequently, the direction of this paper is towards the application and investigation of a more realistic model for the spatial randomness of the APs in terms of a Poisson point process (PPP). Specifically, we derive the downlink coverage probability (CP) of CF mMIMO systems by means of stochastic geometry and deterministic equivalent tools. Notably, it is the only work having derived the CP for CF mMIMO systems. Among the results, we highlight the outperformance of CF mMIMO systems against small cells (SCs), which increases with the density of the APs due to channel hardening, favorable propagation, and interference suppression. Moreover, we observe the saturation of the CP at high AP density.
\end{abstract}

Index Terms-Cell-free massive MIMO systems, heterogeneous networks, coverage probability, stochastic geometry, deterministic equivalents.

\section{INTRODUCTION}

The adoption of massive multiple-input-multiple-output (mMIMO) technology from fifth generation $(5 \mathrm{G})$ networks has been well-grounded [1]. In particular, the mMIMO topology appears with two extremes being a large collocated antenna array in each cell and a large number of geographically distributed antenna arrays [2], [3]. The latter architecture has attracted more attention recently in [3] under the name of cell-free (CF) mMIMO systems by enjoying the benefits of both network MIMO [4] and mMIMO systems [2] such as increased macrodiversity and favorable propagation, respectively. Especially, a large number of access points (APs) serves jointly a smaller number of users by utilizing only local channel state information (CSI) at each AP. Contrary to cellular mMIMO systems, serving poorly the cell-edge users because of the intercell interference, in CF mMIMO systems, the cell boundaries are dissolved as well as the quality of service and expected coverage are improved since the distances between the APs and the users are reduced.

Indisputably, CF mMIMO systems have attracted a lot of scientific interest, e.g., [3], [5]-[9]. Especially, [3] presents the outperformance of CF mMIMO systems against small cells (SCs). In [5], the APs were equipped with multiple antennas towards the study of energy efficiency, while in [6], downlink training was suggested to improve the performance. Other interesting works have considered the limited backhaul, being an obstacle in distributed systems, and a user-centric approach to provide a large achievable rate with reduced backhaul overload in [7] and [8], respectively. Interestingly, [9] studied the assumptions that should hold for favorable propagation and channel hardening by accounting for the spatial randomness of the APs in the analysis.

Disregarding the importance for consideration of the spatial randomness of the APs in CF mMIMO systems due to their highly irregular and opportunistic layout, the majority of works, concerning CF mMIMO systems, has relied on the idealized and inaccurate assumption of uniformly distributed APs in a finite region, e.g., [3]. In fact, this assumption has been taken into account only during the simulation while the analysis lacks this aspect. Note that in the case of SCs, tractable and accurate models, considering the spatial randomness of the base stations by means of stochastic geometry and the theory of Poisson point processes (PPPs), have been presented, for example, in [10]-[12] to model the heterogeneous cell-densification met in $5 \mathrm{G}$ networks.

Following this direction, this work covers the arising need for consideration of the spatial randomness of the APs by a more realistic and accurate model, where the APs are PPP distributed and do not just follow a uniform placement such as in [3]. Moreover, contrary to [9], dealing with mainly the effects of favorable propagation and channel hardening, we focus on the performance of CF mMIMO systems by means of the coverage probability (CP). Specifically, we first derive the downlink deterministic equivalent (DE) of the signal-to-interference-plusnoise ratio (SINR) with conjugate beamforming, and then, the $\mathrm{CP}$ by means of stochastic geometry. Notably, as far as the authors are aware, these are the only analytical results deriving $\mathrm{DE}$ expressions and providing the uplink $\mathrm{CP}$ for $\mathrm{CF}$ mMIMO systems.

The remainder of this paper is structured as follows. Section II presents the system model for CF mMIMO systems with PPP distributed APs. Section III describes the channel estimation phase. In Section IV, we present the downlink transmission and the DE SINR, while Section V provides the CP. Then numerical results follow, and Section VII concludes the paper. 


\section{System MOdeL}

\section{A. Arrangement of APs}

We consider a CF mMIMO systems where a large number of APs each equipped with $N \geq 1$ antennas serves jointly $K$ single-antenna users in the same time-frequency resources. In particular, aiming at realistic results, we assume that the AP locations $\left\{x_{i}\right\} \subset \mathbb{R}^{2}$ are generated randomly in a finitesized geographic area $\mathcal{A}$ of space space $S(\mathcal{A}) \mathrm{m}^{2}$ and follow a two-dimensional homogeneous PPP $\Phi_{\mathrm{AP}}$ with density $\lambda_{\mathrm{AP}}$ $\left[\mathrm{APs} / \mathrm{km}^{2}\right]$. Let $M$ the number of APs in a specific realization of the PPP $\Phi_{\mathrm{AP}}$. Then, $M$ is a Poisson random variable with mean $\tilde{M}=\mathbb{E}[M]$ given by

$$
\tilde{M}=\lambda_{\mathrm{AP}} S(\mathcal{A}) .
$$

In this regard, we denote $\mathcal{W}=M N$ being a Poisson random variable with mean $\mathbb{E}[\mathcal{W}]=\tilde{M} N$ and expressing the total number of antennas in $\mathcal{A}$. The locations of the users are distributed according to some independent stationary point process [13] while their number is obtained by means of a scheduling algorithm allowing the selection of $K$ from a large set of users.

Based on Slivnyak's theorem, we focus on a typical user chosen at random from amongst all users in the network, in order to conduct the analysis and investigate the performance of the network [14]. For the sake of exposition and without any loss of generality, we assume that the typical user is located at the origin and is denoted henceforth by the arbitrary index $k$.

\section{B. Channel Model}

In a specific realization of the PPP $\Phi_{\mathrm{AP}}$ with $M$ APs, let $\mathbf{h}_{m k}$ be the $N \times 1$ channel vector between the $m$ th AP and the typical user which is expressed as

$$
\mathbf{h}_{m k}=l_{m k}^{1 / 2} \mathbf{g}_{m k}, \quad m=1, \ldots, M \text { and } k=1, \ldots, K
$$

with $l_{m k}$ and $\mathbf{g}_{m k}$ representing the independent large-scale and small-scale fadings, respectively. Regarding the large-scale fading, we assume a non-singular bounded pathloss model described by $l_{m k}\left(r_{m k}\right)=\min \left(1, r_{m k}^{-\alpha}\right)$ with $\alpha>0$ being the path-loss exponent and $r_{m k}$ the distance between the $m$ th AP and the typical user [15]. Instead of an unbounded path-loss model such as $l_{m k}\left(r_{m k}\right)=r_{m k}^{-\alpha}$, this choice relies on the fact that the latter would result in unrealistically high power gain in the case of CF massive MIMO systems, where an AP can approach arbitrarily close to a user. Specifically, we assume that the distance $r_{m k}$, actually involving the communication between a random AP and a random user, follows the uniform distribution in $\mathcal{A}$. In addition, the distances from other users are independent and follow the uniform distribution. Concerning, $\mathbf{g}_{m k}$, it models Rayleigh fading, i.e., it consists of smallscale fading elements, which are independent and identically distributed (i.i.d.) $\mathcal{C N}(0,1)$ random variables.

We assume that the coherence time of each coherence interval is $\tau_{\mathrm{c}}=B_{\mathrm{c}} T_{\mathrm{c}}$ samples (channel uses), where $B_{\mathrm{c}}$ in $\mathrm{Hz}$ and $T_{\mathrm{c}}$ in $\mathrm{s}$ denote the coherence bandwidth and time, respectively. In $\tau_{\mathrm{c}}$, we include the uplink training phase of $\tau_{\mathrm{tr}}$ symbols as well as the uplink and downlink data transmission phases of $\tau_{\mathrm{u}}$ and $\tau_{\mathrm{d}}$ samples, respectively. Based on the property of channel reciprocity, being achievable under TDD operation, we assume identical channels for the two data transmission phases.

\section{UPLINK CHANNEL ESTIMATION}

The demand for knowledge of CSI at the transmitter requires an uplink training phase to allow the APs compute the estimates $\hat{\mathrm{g}}_{m k}$ of their local channels. Hence, in each realization of the network, all $K$ users send simultaneously non-orthogonal pilot sequences with duration equal to $\tau_{\mathrm{tr}}<K$ samples due to the limited length of the coherence interval. Note that the subscript tr expresses the training stage. The $N \times \tau_{\text {tr }}$ received channel vector at the $m$ th $\mathrm{AP}$ is given by

$$
\tilde{\mathbf{y}}_{\mathrm{tr}, m}=\sum_{i=1}^{K} \sqrt{\tau_{\operatorname{tr}} \rho_{\mathrm{tr}}} l_{m i}^{1 / 2} \mathbf{g}_{m i} \boldsymbol{\psi}_{i}^{\mathrm{H}}+\mathbf{n}_{\mathrm{tr}, m},
$$

where $\boldsymbol{\psi}_{i} \in \mathbb{C}^{\tau_{\mathrm{tr}} \times 1}$ is the normalized sequence of the $i$ th user with $\left\|\psi_{i}\right\|^{2}=1, \mathbf{n}_{\mathrm{tr}, m}$ is the $N \times \operatorname{tr}$ additive noise vector at the $m$ th AP consisting of i.i.d. $\mathcal{C N}(0,1)$ random elements, and $\rho_{\mathrm{tr}}$ is the normalized signal-to-noise ratio (SNR).

Following the standard procedure in [16] and assuming that the distance $r_{m k}$ is known a priori, the $m$ th AP obtains the minimum mean-squared error (MMSE) estimate as

$$
\begin{aligned}
\hat{\mathbf{h}}_{m k} & =\mathrm{E}\left[\mathbf{h}_{m k}^{\mathrm{H}} \tilde{\mathbf{y}}_{\mathrm{tr}, m k}\right]\left(\mathrm{E}\left[\tilde{\mathbf{y}}_{\mathrm{tr}, m k} \tilde{\mathbf{y}}_{\mathrm{tr}, m k}^{\mathrm{H}}\right]\right)^{-1} \tilde{\mathbf{y}}_{m k} \\
& =\frac{l_{m k}}{\sum_{i=1}^{K}\left|\boldsymbol{\psi}_{i}^{\mathrm{H}} \boldsymbol{\psi}_{k}\right|^{2} l_{m i}+\frac{1}{\tau_{\mathrm{tr}} \rho_{\mathrm{tr}}}} \tilde{\mathbf{y}}_{m k} .
\end{aligned}
$$

while the estimation error vector is given by $\tilde{\mathbf{e}}_{m k}=\mathbf{h}_{m k}-$ $\hat{\mathbf{h}}_{m k}$. The estimated channel and estimation error vectors are uncorrelated and Gaussian distributed and written as $\hat{\mathbf{h}}_{m k} \in \mathbb{C}^{N \times 1} \sim \mathcal{C N}\left(\mathbf{0}, \sigma_{m k}^{2} \mathbf{I}_{N}\right)$ and $\tilde{\mathbf{e}}_{k} \in \mathbb{C}^{N \times 1} \sim$ $\mathcal{C N}\left(\mathbf{0}, \tilde{\sigma}_{m k}^{2} \mathbf{I}_{N}\right)$ since $\mathbf{h}_{m k} \in \mathbb{C}^{N \times 1} \sim \mathcal{C N}\left(\mathbf{0}, l_{m k} \mathbf{I}_{N}\right)$, where $\sigma_{m k}^{2}=\frac{l_{m k}^{2}}{d_{m}}$ and $\tilde{\sigma}_{m k}^{2}=l_{m k}\left(1-\frac{l_{m k}}{d_{m}}\right)$ with $d_{m}=$ $\left(\sum_{i=1}^{K}\left|\boldsymbol{\psi}_{i}^{\mathrm{H}} \boldsymbol{\psi}_{k}\right|^{2} l_{m i}+\frac{1}{\tau_{\mathrm{tr}} \rho_{\mathrm{tr}}}\right)$. At this point, it is better for the sake of following algebraic manipulations to denote the vectors $\mathbf{h}_{k}=\left[\mathbf{h}_{1 k}^{T} \cdots \mathbf{h}_{M k}^{T}\right]^{\top} \in \mathbb{C}^{\mathcal{W} \times 1} \sim \mathcal{C N}\left(\mathbf{0}, \mathbf{L}_{k}\right)$, $\hat{\mathbf{h}}_{k}=\left[\hat{\mathbf{h}}_{1 k}^{T} \cdots \hat{\mathbf{h}}_{M k}^{T}\right]^{\top} \in \mathbb{C}^{\mathcal{W} \times 1} \sim \mathcal{C N}\left(\mathbf{0}, \boldsymbol{\Phi}_{k}\right)$ and $\tilde{\mathbf{e}}_{k} \in$ $\mathbb{C}^{\mathcal{W} \times 1} \sim \mathcal{C} \mathcal{N}\left(\mathbf{0}, \mathbf{L}_{k}-\boldsymbol{\Phi}_{k}\right)$, where the matrices $\mathbf{L}_{k}, \boldsymbol{\Phi}_{k}=$ $\mathbf{L}_{k}^{2} \mathbf{D}^{-1}$, and $\mathbf{D}$ are $\mathcal{W} \times \mathcal{W}$ are block diagonal, i.e., $\mathbf{L}_{k}=$ $\operatorname{diag}\left(l_{1 k} \mathbf{I}_{N}, \ldots, l_{M k} \mathbf{I}_{N}\right), \boldsymbol{\Phi}_{k}=\operatorname{diag}\left(\sigma_{1 k}^{2} \mathbf{I}_{N}, \ldots, \sigma_{M k}^{2} \mathbf{I}_{N}\right)$, and $\mathbf{D}=\operatorname{diag}\left(d_{1} \mathbf{I}_{N} \ldots, d_{M} \mathbf{I}_{N}\right)$, respectively. In addition, we denote $\mathbf{C}_{k}=\boldsymbol{\Phi}_{k}^{-1}$ with $\mathbf{C}_{k}=\operatorname{diag}\left(c_{1 k} \mathbf{I}_{N}, \ldots, c_{M k} \mathbf{I}_{N}\right)$, where $c_{m k}=\sigma_{m k}^{-2}$.

\section{DOWNLINK TRANSMISSION}

The received signal by the typical user is given by

$$
y_{\mathrm{d}, k}=\sqrt{\rho_{\mathrm{d}}} \sum_{i \in \Phi_{\mathrm{AP}}} \tilde{\mathbf{h}}_{i}^{\mathrm{H}} \mathbf{s}_{i}+z_{\mathrm{d}, k},
$$

where $\rho_{\mathrm{d}}$ is the downlink transmit power, $\tilde{\mathbf{h}}_{i}$ is the $N \times 1$ channel vector between the associated AP located at $x_{i} \in \mathbb{R}^{2}$ and the typical user, $\mathbf{s}_{i}$ is the transmit signal from the $i$ th AP, 
and $z_{\mathrm{d}, k} \sim \mathcal{C N}(0,1)$ is the additive Gaussian noise at the $k$ th user.

We can rewrite (5) as

$$
y_{\mathrm{d}, k}=\sqrt{\rho_{\mathrm{d}}} \sum_{m=1}^{M} \mathbf{h}_{m k}^{\mathrm{H}} \mathbf{s}_{m}+z_{\mathrm{d}, k},
$$

since the number of PPP distributed APs in a given realization is $M$. Note that $\mathbf{h}_{m k}$ is the channel between the $m$ th AP and user $k$ while $\mathbf{s}_{m}$ denotes the transmit signal from the $m$ th associated AP, which is given by

$$
\mathbf{s}_{m}=\sqrt{\mu} \sum_{k=1}^{K} \mathbf{f}_{m k} q_{k}
$$

with $q_{k} \in \mathcal{C}$ being the transmit data symbol for the typical user satisfying $\mathbb{E}\left[\left|q_{k}\right|^{2}\right]=1$. Also, $\mathbf{f}_{m k}$ represents the $(m, k)$ th element of a linear precoder, which in this work is selected to be conjugate beamforming due to its computational efficiency and good performance of in both massive MIMO and SCs designs [3], [17]. In particular, the expression of the precoder is $\mathbf{f}_{m k}=c_{m k} \hat{\mathbf{h}}_{m k}$, where the scaling relies on a statistical channel inversion power-control policy that eases the algebraic manipulations henceforth [18]. Moreover, $\mu$, being a normalization parameter, is given by means of the constraint of the transmit power $\mathbb{E}\left[\rho_{\mathrm{d}} \mathbf{S s}^{\mathrm{H}}\right]=\rho_{\mathrm{d}}$, i.e., we have $\mu=\frac{1}{\mathbb{E}\left[\operatorname{tr} \mathbf{F}_{m} \mathbf{F}_{m}^{\mathrm{H}}\right]}$, where $\mathbf{F}_{m}=\left[\mathbf{f}_{m 1} \cdots \mathbf{f}_{m K}\right] \in \mathbb{C}^{N \times K}$ is the precoding matrix.

Overall, the received signal by the typical user can be further written as

$$
\begin{aligned}
& y_{\mathrm{d}, k}=\sqrt{\mu \rho_{\mathrm{d}}} \sum_{m=1}^{M} \sum_{i=1}^{K} c_{m i} \mathbf{h}_{m k}^{\mathrm{H}} \hat{\mathbf{h}}_{m i} q_{i}+z_{\mathrm{d}, k} \\
& =\sqrt{\mu \rho_{\mathrm{d}} \mathbb{E}}\left[\mathbf{h}_{k}^{\mathrm{H}} \mathbf{C}_{k} \hat{\mathbf{h}}_{k}\right] q_{k}+\sqrt{\mu \rho_{\mathrm{d}}} \mathbf{h}_{k}^{\mathrm{H}} \mathbf{C}_{k} \hat{\mathbf{h}}_{k} q_{k} \\
& -\sqrt{\mu \rho_{\mathrm{d}}} \mathbb{E}\left[\mathbf{h}_{k}^{\mathrm{H}} \mathbf{C}_{k} \hat{\mathbf{h}}_{k}\right] q_{k}+\sqrt{\mu \rho_{\mathrm{d}}} \sum_{i \neq k}^{K} \mathbf{h}_{k}^{\mathrm{H}} \mathbf{C}_{i} \hat{\mathbf{h}}_{i} q_{i}+z_{\mathrm{d}, k},
\end{aligned}
$$

where we have used a similar technique to [19] to transform (8) into (9) because user $k$ has knowledge of only $\mathbb{E}\left[\mathbf{h}_{k}^{\mathrm{H}} \mathbf{C}_{k} \hat{\mathbf{h}}_{k}\right]$ and not of the instantaneous CSI. Assuming that (9) represents a single-input single-output (SISO) system, the effective SINR of the downlink transmission from all the APs to the typical user, conditioned on the distances of APs $l_{m k}$ for $m=1, \ldots, M$, is given by

$$
\gamma_{k}=\frac{\left|\mathbb{E}\left[\mathbf{h}_{k}^{\mathrm{H}} \mathbf{C}_{k} \hat{\mathbf{h}}_{k}\right]\right|^{2}}{\operatorname{var}\left[\mathbf{h}_{k}^{\mathrm{H}} \mathbf{C}_{k} \hat{\mathbf{h}}_{k}\right]+\sum_{i \neq k}^{K} \mathbb{E}\left[\left|\mathbf{h}_{k}^{\mathrm{H}} \mathbf{C}_{i} \hat{\mathbf{h}}_{i}\right|^{2}\right]+\frac{1}{\mu \rho_{\mathrm{d}}}},
$$

where the unknown terms have been treated as uncorrelated additive noise. Next, based on the theory of DEs, being a common mathematical tool in the massive MIMO literature [20]-[22], we derive the asymptotic SINR conditioned on the distances of APs as $K, \mathcal{W} \rightarrow \infty$, while the finite ratio $K / \mathcal{W}$ is kept constant such that $\gamma_{k}-\bar{\gamma}_{k} \underset{M \rightarrow \infty}{\stackrel{\text { a.s. }}{\longrightarrow}} 0$, where $\bar{\gamma}_{k}$ is the deterministic SINR and $\underset{n \rightarrow \infty}{\stackrel{\text { a.s. }}{\longrightarrow}} 0$ denotes almost sure convergence.
Proposition 1: Given a realization of $\Phi_{\mathrm{AP}}$ and conditioned on the APs distances, the deterministic SINR of the downlink transmission from the PPP distributed APs to the typical user in a CF massive MIMO system, accounting for pilot contamination and conjugate beamforming, is given by

$$
\bar{\gamma}_{k} \asymp \frac{\mathcal{W}}{\frac{1}{\mathcal{W}} \sum_{i=1}^{K} \operatorname{tr} \mathbf{D L}_{i}^{-2}\left(\mathbf{L}_{k}+\frac{\mathcal{W}}{\rho_{\mathrm{d}}}\right)-1} .
$$

Proof: Herein, we shall omit the proof of Proposition 1, which is provided in [23] due to limited space.

Remark 1: This SINR includes the randomness regarding the AP locations by means of the path-losses between the APs and the users.

Remark 2: The SINR $\bar{\gamma}_{k}$ in 11 saturates in the high SNR regime and with increasing the number of antennas per AP $N$ while it decreases with $K$ and with the severity of pilot contamination.

\section{Coverage Probability}

A typical user is in coverage in a CF massive MIMO system if the downlink SINR from the randomly located APs in the network is higher than the target SINR $T$.

Theorem 1: The downlink CP of a pilot contaminated $\mathrm{CF}$ massive MIMO network, where the APs are PPP distributed and undergo a single-slope path loss model while employing conjugate beamforming, is lower bounded by (12), or equivalently (13) shown at the top of next page, where $\tilde{\mathcal{W}}=\mathbb{E}[\mathcal{W}]$ and $\eta=\tilde{\mathcal{W}}(\tilde{\mathcal{W}} !)^{-\frac{1}{\tilde{\mathcal{W}}}}$.

Proof: See Appendix A.

Remark 3: According to (13), we observe the decrease of $P_{\mathrm{c}}^{\mathrm{cf}}$ with the number of users $K$ and the severity of the pilot contamination. Also, when $T \rightarrow \infty$, the $\mathrm{CP}$ becomes zero while if the path-loss exponent $\alpha>2$ increases, $P_{\mathrm{c}}^{\text {cf }}$ decreases. Moreover, the $\mathrm{CP}$ saturates in the high SNR regime. Concerning the dependence from the AP density and the number of antennas per AP, it is given indirectly by means of $\tilde{\mathcal{W}}$ as a complicated function. Hence, this dependence can be shown only by means of numerical results. In fact, in Sec. VI, it is shown that $P_{\mathrm{c}}^{\mathrm{cf}}$ increases with $\lambda_{\mathrm{AP}}$ and results in a ceiling at large AP density similarly to the case of single-slope path loss models in SCs [24]. The same trend is followed with increasing the number of antennas per AP.

\section{NUMERICAL RESULTS}

Herein, we illustrate the behavior of PPP located APs in a CF architecture by means of the analytical expression of the CP provided by Theorem 1. This graphical representation takes place for the first time in the corresponding literature since prior works have not taken into account for a realistic and well-accepted model for the randomness of APs positions in the analysis.

For the sake of comparison, we consider the system model in [12], denoted as "small cells" or "SCs", where the base stations have the same number of antennas and serve a single user while the imperfect CSI model in that scenario is replaced 


$$
\begin{aligned}
P_{\mathrm{c}}^{\mathrm{cf}} & \geq \sum_{n=1}^{\tilde{\mathcal{W}}}\left(\begin{array}{c}
\tilde{\mathcal{W}} \\
n
\end{array}\right)(-1)^{n+1} e^{-n \eta T\left(\frac{K}{\alpha \pi \rho_{\mathrm{d}}}\left(\sum_{j=1}^{K}\left|\boldsymbol{\psi}_{j}^{\mathrm{H}} \boldsymbol{\psi}_{k}\right|^{2}\left(\alpha \rho_{\mathrm{d}}+\tilde{\mathcal{W}}(\alpha-2)\right)+\frac{(\alpha-2) \rho_{\mathrm{d}}+\tilde{\mathcal{W}}(\alpha-1)}{\tau_{\mathrm{tr}} \rho_{\mathrm{tr}}}\right)-1\right)} \\
& =1-\left(1-e^{-\eta T\left(\frac{K}{\alpha \pi \rho_{\mathrm{d}}}\left(\sum_{j=1}^{K}\left|\boldsymbol{\psi}_{j}^{\mathrm{H}} \boldsymbol{\psi}_{k}\right|^{2}\left(\alpha \rho_{\mathrm{d}}+\tilde{\mathcal{W}}(\alpha-2)\right)+\frac{(\alpha-2) \rho_{\mathrm{d}}+\tilde{\mathcal{W}}(\alpha-1)}{\tau_{\mathrm{tr}} \rho_{\mathrm{tr}}}\right)-1\right)}\right)^{\tilde{\mathcal{W}}} .
\end{aligned}
$$

by the current one and we assume no hardware impairments and channel aging. Therein, independent users are associated with their nearest multi-antenna AP, and the remaining APs play the role of interference. In particular, we consider [12, Th. 1] providing the $\mathrm{CP}$ for SCs. Among the differences between $\mathrm{CF}$ massive MIMO and SCs, we observe that: i) in SCs, the effective channel power does not harden. As a result, SCs require both uplink and downlink training phases but CF massive MIMO systems rely only on uplink training. ii) CF massive MIMO can achieve optimal performance with simple linear processing by enjoying favorable propagation. iii) SCs suffer from inter-cell interference while $\mathrm{CF}$ massive MIMO systems exploit the co-processing among the APs to suppress inter-cell interference by eliminating any cell boundaries. In addition, a fair comparison between $\mathrm{CF}$ massive MIMO systems and SCs requires equal total radiated power in both architectures. Hence, we have that $\bar{\rho}_{\mathrm{tr}}^{\mathrm{sc}}=\bar{\rho}_{\mathrm{tr}}$ and $\bar{p}_{\mathrm{d}}^{\mathrm{sc}}=\frac{M}{K} \bar{p}_{\mathrm{d}}$, where $\bar{\rho}_{\mathrm{tr}}^{\mathrm{sc}}$ and $\bar{p}_{\mathrm{sc}}^{\mathrm{d}}$ are the normalized uplink training and downlink transmit powers [3].

The analytical expressions are verified by means of Monte Carlo simulations represented by black bullets. The simulated results are generated by means of the statistical SINR given by (10) after averaging over $10^{4}$ random instances of the channels while the $\mathrm{CP}$ is obtained as an average of $10^{4}$ realizations of different random AP topologies. Moreover, the results corresponding to CF massive MIMO systems and SCs are depicted by means of "solid" blue and "dot" red lines, respectively. Notably, this is the first work verifying the DE analysis for CF massive MIMO systems.

\section{A. Setup}

A finite window of area of $1 \mathrm{~km} \times 1 \mathrm{~km}$ is chosen with PPP distributed APs having density $\lambda_{\mathrm{AP}}=40 \mathrm{APs} / \mathrm{km}^{2}$ and $N=5$ antennas per AP unless otherwise stated. The area is wrapped around at the edges to prevent any boundary effects. Also, we assume $K=10$ randomly distributed users while the path-loss exponent is $\alpha=3.5$. Actually, similar to [3], we have that the coherence bandwidth and time are $B_{\mathrm{c}}=200 \mathrm{KHz}$ and $T_{\mathrm{c}}=1 \mathrm{~ms}$, respectively. The normalized uplink training transmit power per pilot symbol $\rho_{\text {tr }}$ and downlink transmit power $p_{\mathrm{d}}$ result by dividing $\bar{\rho}^{\text {tr }}$ and $\bar{p}_{\mathrm{d}}$ by the noise power $N_{\mathrm{P}}$ given in $\mathrm{W}$ by $N_{\mathrm{P}}=W_{\mathrm{c}} \times \kappa_{\mathrm{B}} \times T_{0} \times N_{\mathrm{F}}$, where $W_{\mathrm{c}}=20 \mathrm{MHz}, \kappa_{\mathrm{B}}=1.381 \times 10^{-23} \mathrm{~J} / \mathrm{K}, T_{0}=290 \mathrm{~K}$, and $N_{\mathrm{F}}=9 \mathrm{~dB}$. Note that $\rho_{\mathrm{tr}}=100 \mathrm{~mW}$ and $p_{\mathrm{d}}=200 \mathrm{~mW}$ as well as $\tau_{\mathrm{tr}}=\tau_{\mathrm{d}}=10$ samples.

In Fig. 1, we evaluate the performance of the proposed bound by varying the target SINR. The tightness of the proposed bound is very good, but it is relaxed with increasing $\lambda_{\mathrm{AP}}$

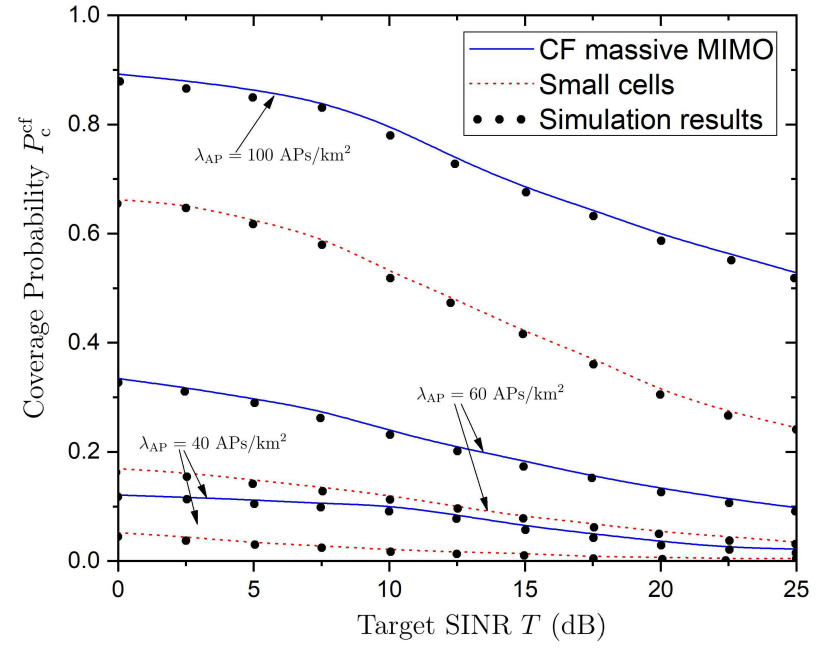

Fig. 1. CP for varying AP density $\lambda_{\mathrm{AP}}$ versus the target SINR $T$ for both CF massive MIMO systems and SCs.

due to the Alzer's inequality. Moreover, Fig. 1 shows the decrease of the CP with $T$ in both cases of CF massive MIMO and SCs because of the inter-user and inter-cell interferences, respectively. Note that when the target SINR tends to zero, the $\mathrm{CP}$ becomes one while when $T \rightarrow \infty, P_{\mathrm{c}}^{\mathrm{sc}}$ approaches zero. However, for typical values of $T$, i.e., around $15 \mathrm{~dB}$, the CP is finite and decreases. Notably, CF massive MIMO systems, unlike SCs, systematically provide higher coverage for all values of the target SINR $T$ because they take benefit from favorable propagation, channel hardening, and suppression of the inter-cell interference. In particular, by increasing the AP density $\lambda_{\mathrm{AP}}$, these effects have a higher contribution to the outperformance of CF massive MIMO systems against SCs.

In Fig. 2, we examine the impact of AP density on the $\mathrm{CP}$ for varying the threshold $T$. This figure illustrates the comparison between CF massive MIMO systems and SCs with respect to their density, which is the fundamental characteristic between the two architectures. Specifically, it is depicted that by increasing $\lambda_{\mathrm{AP}}$ in $\mathrm{CF}$ massive MIMO systems and SCs, $P_{\mathrm{c}}^{\mathrm{sc}}$ increases and saturates at high AP density. Although this behavior is already known for SCs in the case of single-slope path loss models [24], this figure also depicts the performance of CF massive MIMO systems independently and allows the comparison between the two network designs. As can be seen, the coverage by CF massive MIMO systems is higher than SCs as $\lambda_{\mathrm{AP}}$ increases due to favorable propagation and channel hardening met in the former architecture. In 


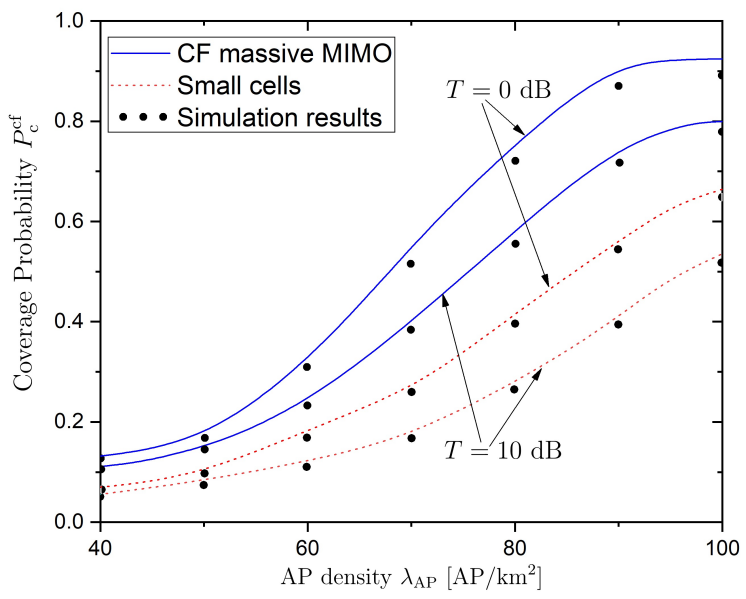

Fig. 2. CP for varying target SINR $T$ versus the AP density $\lambda_{\mathrm{AP}}$ for both $\mathrm{CF}$ massive MIMO systems and SCs.

addition, a higher threshold reduces the $\mathrm{CP}$ since it is less possible to achieve certain coverage at higher values. Moreover, when $\lambda_{\mathrm{AP}}$ increases, the performance gap between the two architectures increases because CF massive MIMO systems are more enhanced by the cooperation among the APs and the massive MIMO property in terms of channel hardening and favorable propagation. Furthermore, the ceiling at high $\lambda_{\mathrm{AP}}$ is equivalent to the independence from $\lambda_{\mathrm{AP}}$ which is known as the SINR invariance described in [24] and Sec. IV of this work for SCs and CF massive MIMO systems, respectively.

\section{CONCLUSION}

In this paper, we proposed a novel framework to model the spatial randomness of APs which has not been accounted adequately before despite the irregularity of their locations in practice. Specifically, this is the unique work exploiting PPP modeling to describe the locations of the APs and has derived a tractable closed-form expression for the $\mathrm{CP}$ of $\mathrm{CF}$ mMIMO systems. Notably, numerical results illustrated that CF mMIMO systems outperform SCs due to the combined benefits from network MIMO and mMIMO systems. Furthermore, the CP increases with the AP density but up to a certain point where it has a ceiling while it decreases with the target SINR.

\section{APPENDIX A}

PROOF OF THEOREM 1

After writing (11) in element-wise form, the DE SINR, conditioned on the distances $r_{m i}$ for $i=1, \ldots, K$, is given by

$$
\bar{\gamma}_{k} \asymp \frac{M N}{\frac{1}{M} \sum_{i=1}^{K} \sum_{m=1}^{M} d_{m} l_{m i}^{-2}\left(l_{m k}+\frac{M N}{\rho_{\mathrm{d}}}\right)-1} .
$$

Substitution of (14) inside the expression of the CP results after several algebraic manipulations in

$$
\begin{aligned}
& \mathbb{P}\left(\bar{\gamma}_{k}>T \mid r_{m 1}, \ldots, r_{m K}\right)=\mathbb{P}\left(\mathcal{W}>T\left(\frac{1}{M} \sum_{i=1}^{K} \sum_{m=1}^{M} \mathcal{I}_{m k}-1\right)\right) \\
& \approx \tilde{\mathbb{P}}\left(\tilde{g}>T\left(\frac{1}{M} \sum_{i=1}^{K} \sum_{m=1}^{M} \mathcal{I}_{m k}-1\right)\right) \\
& \approx 1-\left(1-\exp \left(-\eta T\left(\frac{1}{M} \sum_{i=1}^{K} \sum_{m=1}^{M} \mathcal{I}_{m k}-1\right)\right)\right)^{\tilde{\mathcal{W}}}
\end{aligned}
$$

where we have set $\mathcal{I}_{m k}=d_{m} l_{m i}^{-2}\left(l_{m k}+\frac{M N}{\rho_{\mathrm{d}}}\right)$. By approximating the constant number $\mathcal{W}$ in terms of the dummy gamma variable $\tilde{g}$, having mean $\mathcal{W}=M N$ and shape parameter $\tilde{\mathcal{W}}=\mathbb{E}[W]=\tilde{M} N$, we obtain (15). Note that this approximation becomes tighter as $\tilde{\mathcal{W}}$ goes to infinity [25], i.e., this approximation, used in [26], becomes more accurate in our system model involving a large number of APs. In (16), we have used Alzer's inequality (see [25, Lemma 1]) with $\eta=\tilde{\mathcal{W}}(\tilde{\mathcal{W}} !)^{-\frac{1}{\mathcal{W}}}$. In the next step, we evaluate the expectation with respect to AP locations given that the distances between the APs and the users are uniformly distributed after having applied applied the Binomial theorem. Hence, we have

$$
\begin{aligned}
& P_{\mathrm{c}}^{\mathrm{cf}}=\sum_{n=1}^{\tilde{\mathcal{W}}}\left(\begin{array}{c}
\tilde{\mathcal{W}} \\
n
\end{array}\right)(-1)^{n+1} \mathbb{E}[\exp (-n \eta T(\mathcal{I}-1))] \\
& \geq \sum_{n=1}^{\tilde{\mathcal{W}}}\left(\begin{array}{c}
\tilde{\mathcal{W}} \\
n
\end{array}\right)(-1)^{n+1} e^{n \eta T \lambda_{\mathrm{AP}}} \exp (-n \eta T \mathbb{E}[\mathcal{I}]) \text {, }
\end{aligned}
$$

where we have set $\mathcal{I}=\frac{1}{M} \sum_{i=1}^{K} \sum_{m=1}^{M} \mathcal{I}_{m k}$ and have applied Jensen's inequality since $\exp (\cdot)$ is a convex function. The expectation in (18) is written as

$$
\begin{aligned}
& \lim _{R \rightarrow \infty} \mathbb{E}\left[\frac{1}{M} \sum_{i=1}^{K} \sum_{m=1}^{M} \mathcal{I}_{m k}\right] \\
& =\lim _{R \rightarrow \infty} \mathbb{E}_{M}\left[\mathbb{E}_{\mid M}\left[\frac{1}{M} \sum_{i=1}^{K} \sum_{m \in \Phi_{\mathrm{AP}} \cap B(o, R)}^{M} \mathcal{I}_{m k} \mid M=\Phi(B(o, R))\right]\right] \\
& =\sum_{i=1}^{K} \mathbb{E}\left[\left(\sum_{j=1}^{K}\left|\boldsymbol{\psi}_{j}^{\mathrm{H}} \boldsymbol{\psi}_{k}\right|^{2} l_{m j}+\frac{1}{\tau_{\mathrm{tr}} \rho_{\mathrm{tr}}}\right)\left(l_{m k}+\frac{\mathbb{E}[M] N}{\rho_{\mathrm{d}}}\right) l_{m i}^{-2}\right],
\end{aligned}
$$

where in (19), we have assumed a ball of radius $R$ centered at the origin that contains $M=\Phi(B(o, R))$ points with $S(\mathcal{A})=$ $|B(o, R)|$. By conditioning on this area of radius $R$ and on the number of points in this area, $M$ in the denominator cancels out with the number of points inside the ball. In (20), we have substituted $\mathcal{I}_{m k}$ and $d_{m}$. Then, we result in

$$
\mathcal{I}_{1}=\mathbb{E}\left[\sum_{i=1}^{K}\left(\sum_{j=1}^{K}\left|\boldsymbol{\psi}_{j}^{\mathrm{H}} \boldsymbol{\psi}_{k}\right|^{2} l_{m j}+\frac{1}{\tau_{\mathrm{tr}} \rho_{\mathrm{tr}}}\right) l_{m i}^{-2} l_{m k}\right]
$$


and

$$
\mathcal{I}_{2}=\frac{\lambda_{\mathrm{AP}} N}{\rho_{\mathrm{d}}} \mathbb{E}\left[\sum_{i=1}^{K}\left(\sum_{j=1}^{K}\left|\boldsymbol{\psi}_{j}^{\mathrm{H}} \boldsymbol{\psi}_{k}\right|^{2} l_{m j}+\frac{1}{\tau_{\mathrm{tr}} \rho_{\mathrm{tr}}}\right) l_{m i}^{-2}\right] .
$$

Regarding the first part of (21), we have

$$
\begin{aligned}
& \mathbb{E}\left[\sum_{i=1}^{K} \sum_{j=1}^{K}\left|\boldsymbol{\psi}_{j}^{\mathrm{H}} \boldsymbol{\psi}_{k}\right|^{2} l_{m j} l_{m i}^{-2} l_{m k}\right] \\
& = \begin{cases}\sum_{i=1}^{K}\left|\boldsymbol{\psi}_{i}^{\mathrm{H}} \boldsymbol{\psi}_{k}\right|^{2} \mathbb{E}\left[l_{m i}^{-1} l_{m k}\right] & \text { if } j=i \\
\sum_{i=1}^{K} \mathbb{E}\left[l_{m i}^{-2} l_{m k}^{2}\right] & \text { if } j=k \\
\sum_{j \neq i, k}^{K}\left|\boldsymbol{\psi}_{j}^{\mathrm{H}} \boldsymbol{\psi}_{k}\right|^{2} \mathbb{E}\left[l_{m j} l_{m i}^{-2} l_{m k}\right] & \text { otherwise }\end{cases}
\end{aligned}
$$

The expectation in the first branch of (23) for $i \neq k$ gives

$$
\begin{aligned}
\mathbb{E}\left[l_{m i}^{-1} l_{m k}\right] & \geq \frac{1}{\mathbb{E}\left[l_{m i}\right]} \mathbb{E}\left[l_{m k}\right] \\
& =1,
\end{aligned}
$$

where (24) takes advantage of Jensen's inequality, and then, (25) is obtained since the two variables have the same marginal distribution. Similar steps can be followed for the second branch while the last branch becomes

$$
\mathbb{E}\left[l_{m j} l_{m i}^{-2} l_{m k}\right]=\left\{\begin{array}{ll}
\mathbb{E}\left[l_{m j} l_{m k}^{-1}\right] & \text { if } i=k \\
\mathbb{E}\left[l_{m j} l_{m i}^{-2} l_{m k}\right] & \text { if } i \neq k
\end{array} .\right.
$$

If $i=k$, the expression in the first branch is identical to (24) The remaining term in (26) is written as

$$
\begin{aligned}
\mathbb{E}\left[l_{m j} l_{m i}^{-2} l_{m k}\right] & =\mathbb{E}\left[l_{m j}\right] \mathbb{E}\left[l_{m i}^{-2}\right] \mathbb{E}\left[l_{m k}\right] \\
& \geq \mathbb{E}\left[l_{m j}\right] \mathbb{E}\left[l_{m i}^{-1}\right]^{2} \mathbb{E}\left[l_{m k}\right] \\
& \geq 1,
\end{aligned}
$$

where (27) considers the independence among the variables, while (28) exploits the inequality $\mathbb{E}\left[x^{2}\right] \geq \mathbb{E}[x]^{2}$. Last, (29) follows basically the same steps as those taken in (25). The second part of (21) becomes

$$
\mathbb{E}\left[\sum_{i=1}^{K} l_{m i}^{-2} l_{m k}\right]=\left\{\begin{array}{ll}
\mathbb{E}\left[l_{m i}^{-1}\right] & \text { if } i=k \\
\sum_{i \neq k}^{K} \mathbb{E}\left[l_{m i}^{-2} l_{m k}\right] & \text { if } i \neq k
\end{array} .\right.
$$

The first branch i.e., $\mathbb{E}\left[l_{m i}^{-v}\right]$ for $v=1$ results in

$$
\mathbb{E}\left[l_{m i}^{-v}\right] \geq \frac{1}{\mathbb{E}\left[l_{m i}^{v}\right]},
$$

where Jensen's inequality has been applied in (31). The final expression is obtained by computing $\mathbb{E}\left[l_{m i}^{v}\right]$ as

$$
\begin{aligned}
\mathbb{E}\left[l_{m i}^{v}\right] & =2 \pi\left(\int_{0}^{1} y \mathrm{~d} y+\int_{1}^{\infty} y^{-v a+1} \mathrm{~d} y\right) \\
& =\frac{v \alpha \pi}{v \alpha-2} .
\end{aligned}
$$

The second expectation in (30) is computed as

$$
\mathbb{E}\left[l_{m i}^{-2} l_{m k}\right]=\frac{\alpha-2}{\alpha \pi},
$$

where we have used similar techniques as before. By substituting all these expressions in (21), we obtain $\mathcal{I}_{1}$. Similarly, $\mathcal{I}_{2}$ is obtained. Substitution of their expressions in (20) completes the proof. Note that (17) results after using the binomial theorem.

\section{REFERENCES}

[1] C. Wang et al., "A survey of 5G channel measurements and models," IEEE Commun. Surveys Tuts., vol. 20, no. 4, pp. 3142-3168, Fourthquarter 2018.

[2] E. Larsson et al., "Massive MIMO for next generation wireless systems," IEEE Commun. Mag., vol. 52, no. 2, pp. 186-195, February 2014.

[3] H. Q. Ngo et al., "Cell-free massive MIMO versus small cells," IEEE Trans. Wireless Commun., vol. 16, no. 3, pp. 1834-1850, 2017.

[4] S. Shamai and B. M. Zaidel, "Enhancing the cellular downlink capacity via co-processing at the transmitting end," in IEEE VTS 53rd Vehicular Technology Conference, VTC 2001 Spring, vol. 3, pp. 1745-1749.

[5] H. Q. Ngo et al., "On the total energy efficiency of cell-free massive MIMO," IEEE Trans. Green Commun. and Net., vol. 2, no. 1, pp. 25-39, 2018.

[6] G. Interdonato et al., "How much do downlink pilots improve cellfree massive MIMO?" in IEEE Global Communications Conference (GLOBECOM). IEEE, 2016, pp. 1-7.

[7] M. Bashar et al., "Cell-free massive MIMO with limited backhaul," in IEEE International Conference on Communications (ICC), May 2018, pp. 1-7.

[8] S. Buzzi and C. D'Andrea, "Cell-free massive MIMO: User-centric approach," IEEE Wireless Commun. Lett., vol. 6, no. 6, pp. 706-709, Dec 2017.

[9] Z. Chen and E. Björnson, "Channel hardening and favorable propagation in cell-free massive MIMO with stochastic geometry," IEEE Trans. Commun., vol. 66, no. 11, pp. 5205-5219, 2018.

[10] J. G. Andrews, F. Baccelli, and R. K. Ganti, "A tractable approach to coverage and rate in cellular networks," IEEE Trans. Commun., vol. 59, no. 11 , pp. 3122-3134, 2011.

[11] H. S. Dhillon, M. Kountouris, and J. G. Andrews, "Downlink MIMO HetNets: Modeling, ordering results and performance analysis," IEEE Trans. Wireless Commun., vol. 12, no. 10, pp. 5208-5222, 2013.

[12] A. Papazafeiropoulos and T. Ratnarajah, "Towards a realistic assessment of multiple antenna HCNs: Residual additive transceiver hardware impairments and channel aging," IEEE Trans. Veh. Tech., vol. 66, no. 10, pp. 9061-9073, Oct 2017.

[13] M. Kountouris and J. G. Andrews, "Downlink SDMA with limited feedback in interference-limited wireless networks," IEEE Trans. Wireless Commun., vol. 11, no. 8, pp. 2730-2741, 2012.

[14] S. N. Chiu et al., Stochastic geometry and its applications. John Wiley \& Sons, 2013.

[15] M. Haenggi, R. K. Ganti et al., "Interference in large wireless networks," Foundations and Trends $\AA$ in Networking, vol. 3, no. 2, pp. 127-248, 2009.

[16] S. Verdú, Multiuser detection. Cambridge university press, 1998.

[17] T. L. Marzetta et al., Fundamentals of Massive MIMO. Cambridge University Press, 2016.

[18] E. Björnson, L. Sanguinetti, and M. Kountouris, "Deploying dense networks for maximal energy efficiency: Small cells meet massive MIMO," IEEE J. Sel. Areas Commun., vol. 34, no. 4, pp. 832-847, 2016.

[19] M. Medard, "The effect upon channel capacity in wireless communications of perfect and imperfect knowledge of the channel," IEEE Trans. Inf. Theory, vol. 46, no. 3, pp. 933-946, May 2000.

[20] W. Hachem et al., "Deterministic equivalents for certain functionals of large random matrices," The Annals of Applied Probability, vol. 17, no. 3, pp. 875-930, 2007.

[21] R. Couillet and M. Debbah, Random matrix methods for wireless communications. Cambridge University Press, 2011.

[22] A. K. Papazafeiropoulos and T. Ratnarajah, "Deterministic equivalent performance analysis of time-varying massive MIMO systems," IEEE Trans. Wireless Commun., vol. 14, no. 10, pp. 5795-5809, 2015.

[23] A. K. Papazafeiropoulos et al., "Coverage probability of cell-free massive MIMO systems," IEEE Trans. Veh. Tech., accepted with minor revision, available upon request, 2019.

[24] J. G. Andrews et al., "Are we approaching the fundamental limits of wireless network densification?" IEEE Commun. Mag., vol. 54, no. 10, pp. 184-190, 2016.

[25] H. Alzer, "On some inequalities for the incomplete gamma function," Mathematics of Computation of the American Mathematical Society, vol. 66, no. 218, pp. 771-778, 1997.

[26] T. Bai and R. W. Heath, "Analyzing uplink SINR and rate in massive MIMO systems using stochastic geometry," IEEE Trans. Commun., vol. 64, no. 11, pp. 4592-4606, Nov 2016. 